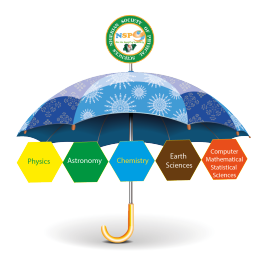

\title{
Exponentially Fitted Chebyshev Based Algorithm as Second Order Initial Value Solver
}

\author{
E. O. Adeyefa ${ }^{a, *}$, O. S. Esan ${ }^{\mathrm{a}}$ \\ ${ }^{a}$ Mathematics Department, Faculty of Science, Federal University Oye-Ekiti, Ekiti State, Nigeria
}

\begin{abstract}
In this research work, we focus on development of a numerical algorithm which is well suited as integrator of initial value problems of order two. Exponential function is fitted into the Chebyshev polynomials for the formulation of this new numerical integrator. The efficiency, ingenuity and computational reliability of any numerical integrator are determined by investigating the zero stability, consistency and convergence of the integrator. Findings reveal that this algorithm is convergent. On comparison, the solutions obtained through the algorithm compare favourably well with the analytical solutions.
\end{abstract}

Keywords: Chebyshev polynomial, Convergence, IVPs, Numerical Integrator, ODEs

Article History :

Received: 10 August 2019

Received in revised form: 23 January 2020

Accepted for publication: 19 February 2020

Published: 05 March 2020

(C)2020 Journal of the Nigerian Society of Physical Sciences. All rights reserved. Communicated by: B. J. Falaye

\section{Introduction}

The search for numerical methods to accurately integrate ordinary differential equation is on the increase and of recent, much effort has been concentrated on solving initial and boundary value problems. Variety of tools (polynomials) have been employed to develop numerical methods for integrating mathematical problems and modelling, an important perspective which cuts across all kind of mathematical problems.

Butcher [1], Lambert [2, 3] and Henrici [4] discussed extensively the approach of reducing higher order ODEs to a system of lower order, specifically, order one and then applying various methods available for solving the resulting system of rst order IVPs. The increase in the number of equations resulting from this approach has been reported as its major setback (see [5, 6]). Jator and Li [7] formulated block methods which directly solve higher order ordinary differential equations without reducing the ODEs to system of first order equations. Researchers such as [8-11] modelled series of algorithms which on their implementation, solve first order initial value problems. Enoch and Ibijola [12] developed a self-adjusting numerical integrator with an inbuilt switch for discontinuous initial value problems.

The aim of developing new methods has always been to introduce a new approach with a target to reduce the error of approximation and thereby improve on the accuracy and efficiency of existing methods hence, this research paper. In this paper, we consider Chebyshev polynomial owing to its elegant properties whereby exponential function shall be fitted into Chebyshev polynomials to develop a direct integrator of second order IVPs. 


\section{Formulation of Exponentially fitted Chebyshev Method}

This section describes the formulation of an algorithm which directly integrate second order initial value problems. We set out by considering a function of the form

$$
f(x)=a_{n} T_{n}(x)+e^{x}
$$

where $T_{n}(x)$ is a Chebyshev polynomial of first degree, $e^{x}$ is exponential function and $a_{n}$ is a constant to be determined in the interval $[a, b], x_{n}=a+n h, n=0,1,2, \ldots$ and $h$ is the step size. We consider $y_{n}$ as numerical estimate to the theoretical value $y\left(x_{n}\right)$ and $f_{n}$ represents $f\left(x_{n}, y_{n}\right)$ with assumption that the theoretical solution $y(x)$ of a given ordinary differential equation can be locally represented in the interval $\left[x_{n}, x_{n+1}\right]$ by the interpolating function described above. Considering $T_{n}(x)$ in equation 1 for $n=0,1,2, \ldots$, we obtain

$$
f(x)=a_{0}+a_{1} x+a_{2}\left(2 x^{2}-1\right)+e^{x}
$$

where $a_{0}, a_{1}$ and $a_{2}$ are constants and also real undetermined coefficient. Interpolating equation 2 at point $x$, we obtain, for $x=x_{n}$

$$
f\left(x_{n}\right)=a_{0}+a_{1} x_{n}+a_{2}\left(2 x_{n}^{2}-1\right)+e^{x_{n}} \equiv y_{n}
$$

and for $x=x_{n+1}$

$$
\begin{array}{r}
f\left(x_{n+1}\right)=a_{0}+a_{1}\left(x_{n}+h\right)+a_{2}\left(2 \left(x_{n}+\right.\right. \\
\left.h)^{2}-1\right)+e^{x_{n}+h} \equiv y_{n+1}
\end{array}
$$

where $x_{n+1}=x_{n}+h$

Differentiating the interpolating function with respect to $x$ and obtaining the first, second and third derivatives of the function, we have

$$
\begin{aligned}
& F^{\prime}\left(x_{n}\right)=a_{1}+4 a_{2} x+e^{x}=f_{n} \\
& F^{\prime \prime}\left(x_{n}\right)=4 a_{2}+e^{x}=f_{n}^{\prime} \\
& F^{\prime \prime \prime}\left(x_{n}\right)=e^{x}=f_{n}^{\prime \prime}
\end{aligned}
$$

Thus, equations 5, 6 and 7 become

$$
\begin{aligned}
& a_{1}+4 a_{2} x+e^{x}=f_{n} \equiv d_{1} \\
& 4 a_{2}+e^{x}=f_{n}^{\prime} \equiv d_{2} \\
& e^{x}=f_{n}^{\prime \prime} \equiv d_{3}
\end{aligned}
$$

Subtracting equation 3 from equation 4 and simplifying yields

$$
\begin{array}{r}
Y=\quad a_{1}\left(x_{n}+h\right)+a_{2}\left(2\left(x_{n}+h\right)^{2}-1\right)+ \\
e^{x_{n}+h}-a_{1} x_{n}-a_{2}\left(2 x_{n}^{2}-1\right)-e^{x_{n}}
\end{array}
$$

where $Y=y_{n+1}-y_{n}$, which gives

$$
y_{n+1}=y_{n}+a_{1}\left(x_{n}+h\right)+a_{2}\left(2\left(x_{n}+h\right)^{2}-1\right)+
$$

$$
e^{x_{n}+h}-a_{1} x_{n}-a_{2}\left(2 x_{n}^{2}-1\right)-e^{x_{n}}
$$

Solving equations 8 and 9, the values coefficients a's are obtained and substituted in equation 2 to have

$$
\begin{gathered}
y_{n+1}=y_{n}+\left(d_{1}-\left(d_{2}-e^{x}\right) x-e^{x}\right)\left(x_{n}+h\right)+ \\
\frac{1}{4}\left(d_{2}-x^{x}\right)\left(2\left(x_{n}+h\right)^{2}-1\right)+ \\
e^{x_{n}+h}-\left(d_{1}-\left(d_{2}-e^{x}\right) x-e^{x}\right) x- \\
\left.e^{x}\right) x_{n}-\frac{1}{4}\left(d_{2}-e^{x}\right) \\
\left(2 x_{n}^{2}-1\right)-e^{x_{n}}
\end{gathered}
$$

Equation 13 is therefore the new numerical integrator for integrating second order initial value problems.

\section{Analysis of the Method}

The convergence of the scheme is investigated using Lipschitz Continuity theorem as discussed by Fatunla [13, 14] and Lambert [2].

Consider $f(x, y)-f\left(x, y^{*}\right)=\frac{\partial f(x, y)}{\partial y}\left(x, y^{*}\right)$ and $L=\sup _{(x, y) \in D o m} \frac{\partial f(x, y)}{\partial y}$ where $f(x, y)$ is defined for all points $(x, y)$ in the region $D=$ $\left\{(x, y) \mid x_{o} \leq x \leq x_{n},-\infty<y<\infty\right\}, x_{o}$ and $x_{n}$ are finite and $L$ is Lipschitz constant such that for $L \leq 0$,

$$
\begin{gathered}
\left|f(x, y)-f\left(x, y^{*}\right)\right| \leq L\left|y-y^{*}\right| \\
f(x, y)-f\left(x, y^{*}\right)=\frac{\partial f(x, y)}{\partial y}
\end{gathered}
$$

Equation 14 can be satisfied if we choose

$$
L=\sup \frac{\partial f(x, y)}{\partial y}
$$

From equation 13 that is, the numerical scheme developed, we have

$$
\begin{aligned}
y_{n+1}=y_{n} & +\left(d_{1}-4\left(\frac{1}{4} d_{2}-\frac{1}{4} e^{x}\right) x-e^{x}\right)\left(x_{n}+h\right) \\
+ & \left(\frac{1}{4} d_{2}-\frac{1}{4} e^{x}\right)\left(2\left(x_{n}+h\right)^{2}-1\right)+e^{x_{n}+h} \\
& -\left(d_{1}-4\left(\frac{1}{4} d_{2}-\frac{1}{4} e^{x}\right) x-e^{x}\right) x_{n}- \\
& \left(\frac{1}{4} d_{2}-\frac{1}{4} e^{x}\right)\left(2 x_{n}^{2}-1\right)-e^{x_{n}}
\end{aligned}
$$

By Lambert [2] and Fatunla [13] as mentioned above,

$$
\begin{aligned}
f(x, y)-f\left(x, y^{*}\right) & =\frac{\partial f(x, y)}{\partial y} \\
L & =\sup _{(x, y) \in \operatorname{Dom}} \frac{\partial f(x, y)}{\partial y}
\end{aligned}
$$

By Henrici [9], $\left|\phi\left(x, y^{*} ; h\right)-\phi(x, y ; h)\right| \leq L\left|y^{*}-y\right|$ where $x \in$ $(a, b), y \in(-\infty, \infty), a \leq h \leq h_{o} ; h_{o}>0$. From equation 14,

$$
\begin{array}{r}
y_{n+1}=\quad y_{n}+\left(d_{1}-4\left(\frac{1}{4} d_{2}-\frac{1}{4} e^{x}\right) x-e^{x}\right)\left(x_{n}+h\right) \\
+\left(\frac{1}{4} d_{2}-\frac{1}{4} e^{x}\right)\left(2\left(x_{n}+h\right)^{2}-1\right)+e^{x_{n}+h}
\end{array}
$$




$$
\begin{gathered}
-\left(d_{1}-4\left(\frac{1}{4} d_{2}-\frac{1}{4} e^{x}\right) x-e^{x}\right) x_{n}- \\
\left(\frac{1}{4} d_{2}-\frac{1}{4} e^{x}\right)\left(2 x_{n}^{2}-1\right)-e^{x_{n}}
\end{gathered}
$$

This is further simplified to have

$$
\begin{array}{rc}
y_{n+1}= & y_{n}+h\left[\left(d_{2}-e^{x}\right) x+e^{x}-d_{1}+\right. \\
& \left.\frac{1}{4}\left(d_{2}-e^{x}\right)(2 h+4 x)\right]+e^{x+h}-e^{x}
\end{array}
$$

Recall that

$$
\begin{aligned}
& d_{1}=F(x, y) \\
& d_{2}=F^{\prime}(x, y)
\end{aligned}
$$

Substituting equations 19 and 20 into 18 and simplifying the resulting equation, we have

$$
\begin{gathered}
y_{n+1}=y_{n}+h\left[2\left(x+\frac{h}{2}\right)\left(F^{\prime}(x, y)-e^{x}\right)+\right. \\
\left.e^{x}-F(x, y)\right]+e^{x+h}-e^{x}
\end{gathered}
$$

We write the functional dependence $y_{n+1}$ on the quantities $x_{n}$, $y_{n}$ and $h$ in the form

$$
\begin{gathered}
\phi\left(x_{n}, y_{n} ; h\right)=\left[\left(F^{\prime}(x, y)-e^{x}\right)\left(2 x+\frac{h}{2}\right)-\right. \\
\left.F(x, y)+e^{x}\right] \\
\phi\left(x_{n}, y_{n}^{*} ; h\right)=\left[\left(F^{\prime}\left(x, y^{*}\right)-e^{x}\right)\left(2 x+\frac{h}{2}\right)-\right. \\
\left.F\left(x, y^{*}\right)+e^{x}\right]
\end{gathered}
$$

$$
\begin{gathered}
\phi\left(x_{n}, y_{n} ; h\right)-\quad \phi\left(x_{n}, y_{n}^{*} ; h\right)=\left[\left(F^{\prime}(x, y)-e^{x}\right)\right. \\
\left.\left(2 x+\frac{h}{2}\right)-F(x, y)+e^{x}\right]- \\
{\left[\left(F^{\prime}\left(x, y^{*}\right)-e^{x}\right)\right.} \\
\left.\left(2 x+\frac{h}{2}\right)-F\left(x, y^{*}\right)+e^{x}\right]
\end{gathered}
$$

$$
\begin{gathered}
\phi\left(x_{n}, y_{n} ; h\right)-\quad \phi\left(x_{n}, y_{n}^{*} ; h\right)=\left[\left[\left(F^{\prime}\left(x, y^{*}\right)-e^{x}\right)\right.\right. \\
]-\left(F^{\prime}(x, y)-e^{x}\right)\right]\left(2 x+\frac{h}{2}\right)+ \\
{\left[F\left(x, y^{*}\right)-F(x, y)\right]}
\end{gathered}
$$

$y_{n+1}=y_{n}+h \phi\left(x_{n}, y_{n} ; h\right)$

Applying equation 17, we have

$$
\begin{aligned}
& F\left(x_{n}, y_{n}^{*}\right)-F\left(x_{n}, y_{n}\right)=\frac{\partial F\left(x_{n}, y_{n}\right)}{\partial y}\left(y_{n}^{*}-y_{n}\right) \\
& F^{\prime}\left(x_{n}, y_{n}^{*}\right)-F^{\prime}\left(x_{n}, y_{n}\right)=\frac{\partial F^{\prime}\left(x_{n}, y_{n}\right)}{\partial y}\left(y_{n}^{*}-y_{n}\right)
\end{aligned}
$$

where $L_{1}=\sup _{(x, y) \in \operatorname{Dom}} \frac{\partial F\left(x_{n}, y_{n}\right)}{\partial y_{n}}$ and $L_{2}=\sup _{(x, y) \in \operatorname{Dom}} \frac{\partial F^{\prime}\left(x_{n}, y_{n}\right)}{\partial y_{n}}$

Putting equations 25 and 26 into 24, we have

$$
\phi\left(x_{n}, y_{n} ; h\right)-\phi\left(x_{n}, y_{n}^{*} ; h\right)=\left[\frac{\partial F^{\prime}\left(x_{n}, y\right)}{\partial y}\left(y_{n}^{*}-y_{n}\right)\right.
$$

$$
\begin{gathered}
\left(2 x+\frac{h}{2}\right]+\frac{\partial F\left(x_{n}, y\right)}{\partial y}\left(y_{n}^{*}-y_{n}\right) \\
{\left[F\left(x, y^{*}\right)-F(x, y)\right]}
\end{gathered}
$$

Putting $L_{1}$ and $L_{2}$ into equation 27 and letting $P=\left(2 x+\frac{h}{2}\right)$, we obtain

$$
\phi\left(x_{n}, y_{n}^{*} ; h\right)-\phi\left(x_{n}, y_{n}^{*} ; h\right)=\left[\left(L_{1}+P L_{2}\right)\left(y_{n}^{*}-y_{n}\right)\right]
$$

If $L_{1}+P L_{2}=L$, equation 28 becomes $\phi\left(x_{n}, y_{n}^{*} ; h\right)-\phi\left(x_{n}, y_{n}^{*} ; h\right)=$ $L\left(y_{n}^{*}-y_{n}\right)$ and $\left|\phi\left(x_{n}, y_{n}^{*} ; h\right)-\phi\left(x_{n}, y_{n}^{*} ; h\right)\right| \leq L\left|y_{n}^{*}-y_{n}\right|$. This shows clearly that the numerical scheme is convergent as it satisfies the Lipschitz condition.

\section{Numerical Experiments}

Two test problems are considered here to implement the derived scheme.

\section{Example 1:}

$$
y^{\prime \prime}=-\frac{6}{x} y^{\prime}-\frac{4}{x^{2}} y, \quad y(1)=1, y^{\prime}=1 ; h=\frac{0.1}{32} .
$$

Exact solution:

$$
y(x)=\frac{5}{3 x}-\frac{2}{3 x^{4}} .
$$

\section{Example 2:}

$$
y^{\prime \prime}=-y^{\prime}, \quad y(0)=1, y^{\prime}(0)=1, h=0.001 .
$$

Exact solution:

$$
y(x)=\cos x .
$$

The graphical solutions of the examples above are shown below.

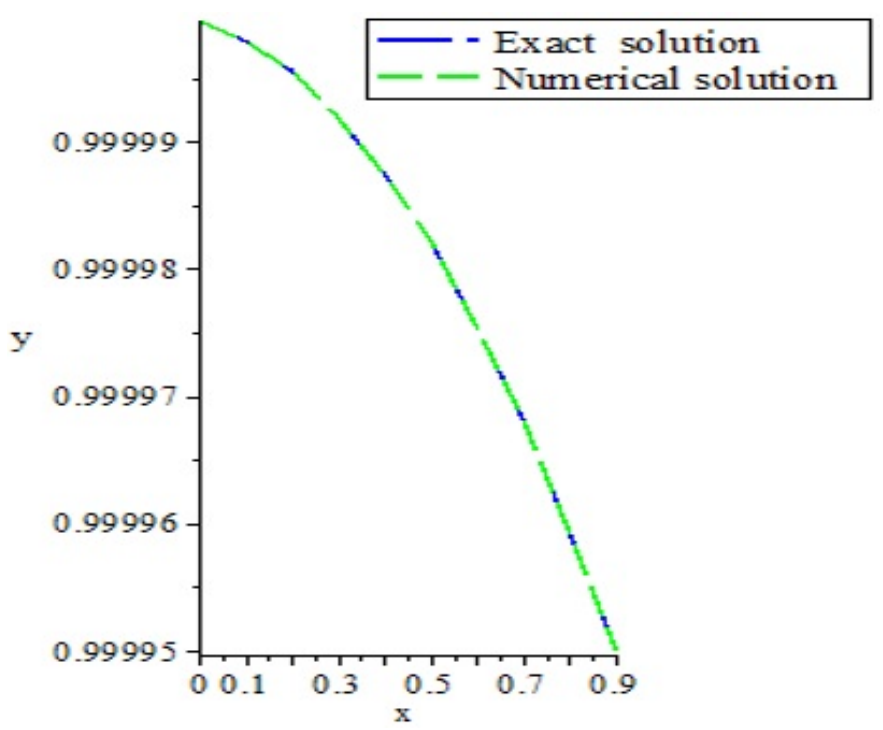

Figure 1: Comparison of the exact and numerical solutions for example 1.

\subsection{Discussion of Results}

We implement the constructed scheme on the two examples considered in this work. In Figures 1 and 2, we compare the 


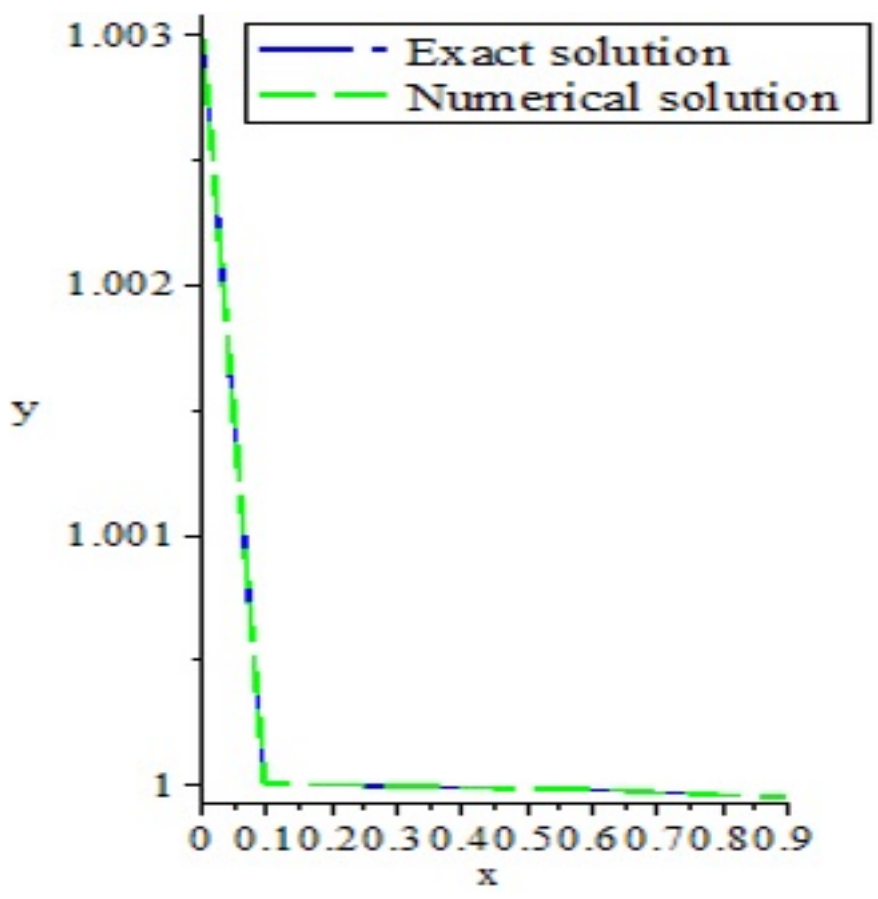

Figure 2: Comparison of the exact and numerical solutions for example 2.

accuracy of the proposed method with the exact solution. From the graphs displayed, it is evident that the new scheme compares favourably with the exact solutions.

\section{Conclusion}

We have developed a scheme to solve second order Initial Value Problems in Ordinary Differential Equations (ODE) using Chebyshev polynomials with exponential function fitted into it. Formulation of numerical integrator using the generated polynomials has been demonstrated. The scheme has been implemented using two test problems. On comparison, the solutions obtained through the numerical scheme recovers the analytical solutions. We therefore recommend the technique for numerical algorithm as we hope to extend the approach to solve boundary value problems.

\section{Acknowledgments}

We thank the referees for the positive enlightening comments and suggestions, which have greatly helped us in making improvements to this paper.

\section{References}

[1] J. C. Butcher "A modified Multistep Method for the Numerical Integration of Ordinary Differential Equations", Journal of the ACM, 12 (1965) 124.

[2] J. D. Lambert "Computational methods in Ordinary differential system", John Wiley, New York (1973).

[3] J. D. Lambert "Numerical Methods for Ordinary Differential Systems", John Wiley, New York (1991).

[4] P. Henrici "Discrete Variable Methods in ODE", New York, John Wiley and Sons (1962).

[5] E. O. Adeyefa "Orthogonal-based hybrid block method for solving general second order initial value problems", Italian journal of pure and applied mathematics 37 (2017) 659.

[6] S. N. Jator \& E. O. Adeyefa "Direct Integration of Fourth Order Initial and Boundary Value Problems using Nystrom Type Methods", IAENG International Journal of Applied Mathematics 49 (2019) 638.

[7] S. N Jator \& J. Li "A self-starting linear multistep method for a direct solution of the general second order initial value problem", International Journal of Computational Mathematics 86 (2009) 827.

[8] E. A. Ibijola "New Schemes for Numerical Integration of Special Initial Value Problems in Ordinary Differential Equations", Ph.d Thesis, University of Benin, Nigeria (1997).

[9] E. A. Ibijola \& P. Kama "On the convergence, consistency and stability of A new One-Step Method for Numerical integration of ODE", International Journal of Computational Mathematics 73 (1999) 261.

[10] O. O. Enoch \& A. A. Olatunji: "A New Self-Adjusting Numerical Integrator for the Numerical Solutions of Ordinary Differential Equations", Global Journal GJSFR 12 (2012) 1022.

[11] S. O. Ayinde \& E. A. Ibijola "A new numerical method for solving first order differential equations", American journal of applied mathematics and statistics 3 (2015) 156.

[12] O. O. Enoch \& E. A. Ibijola "A Self-Adjusting Numerical Integrator with an inbuilt Switch for Discontinuous Initial Value Problems", Australian Journal of Basic and Applied Sciences 5 (2011) 1560.

[13] S. O. Fatunla "Numerical methods for IVPs, in ODEs", Academic press. USA (1988)

[14] S. O. Fatunla "A new Algorithm for numerical solution of ODEs. Computer and Mathematics with Applications", 2 (1976) 247. 